Article

\title{
Phase Behavior of Copolymers Confined in Multi-Walled Nanotubes: Insights from Simulations
}

\author{
Yuxin Zuo ${ }^{1}$, Guoqiang Wang ${ }^{1}$, Ying Yu ${ }^{1, *}$, Chuncheng Zuo ${ }^{1,2}$, Luyou Shi ${ }^{1}$, Fulei Shi ${ }^{1}$ \\ and Jie Wei ${ }^{1}$
}

1 College of Mechanical Science and Engineering, Jilin University, Changchun 130022, China; E-Mails: yuxinzuo_jlu@163.com (Y.Z.);wgq@jlu.edu.cn (G.W.); zuocc@jlu.edu.cn (C.Z.); sluyou@163.com (L.S.); shifl12@mails.jlu.edu.cn (F.S.); weijie12@mails.jlu.edu.cn (J.W.)

2 College of Mechanical and Electrical Engineering, Jiaxing University, Jiaxing 314033, China

* Author to whom correspondence should be addressed; E-Mail: yingyu_jlu@163.com; Tel./Fax: +86-431-8509-5078.

Academic Editor: Philipp Vana

Received: 13 September 2014 / Accepted: 7 January 2015 / Published: 16 January 2015

\begin{abstract}
In this paper, the self-assembly process of diblock copolymers confined in multi-walled cylindrical nanotubes is systematically investigated using a molecular dynamics (MD) method. The dependence of resultant morphologies on the degree of confinement and on the interaction strength between nanotubes and copolymers is studied comprehensively. When the wall surfaces are not preferential, results indicate that geometric confinement significantly influences copolymer conformations. In addition, the thickness of the helical lamellar structure increases with interaction strength and confinement size. In cases where the nanotubes are strongly attracted to one copolymer block, the confinement effect weakens as geometric space increases. Findings explain the dependence of chain conformation on the degree of confinement and the strength of surface preferences.
\end{abstract}

Keywords: self-assembly; block copolymers; multi-walled nanotubes; phase behavior; molecular dynamics 


\section{Introduction}

Block copolymers are macromolecules that consist of two or more chemically immiscible sub-chains or blocks. The mutual repulsion of different blocks and the packing constraints imposed by the connectivity of each block form a self-assembly of rich variables of ordered structures dependent on the number of different blocks, block compositions, and chain architectures (linear, branched, or star-like). Self-assembly processes are driven by forces that direct block copolymers toward whichever minimum equilibrium energy configuration exhibits an appropriate degree of order [1]. The self-assembled structures of block copolymers continue to be the focus of intense research due to demand for fabricated nanomaterials associated with the rapid development of nanoscience. These microscopic ordered structures show great potential for technological application in a wide range of fields, from materials science to biology [2-7]. Potential applications specifically include optical devices [8]; surfaces with molecular recognition capabilities [9]; and templates for nanowire fabrication, nanolithography, and high-density magnetic data storage media [10]. To this effect, building a better understanding the controlling factors of self-assembly system morphologies is important. Controlling the morphology and positioning of block copolymers at appropriate locations in nanostructures is essential to achieve desired properties [11].

Geometrical confinements of block copolymers are realized by placing these copolymers inside a confining environment. Confinements serve as efficient routes for fabricating and controlling molecular organization through the commensurability between the physical dimension of the confinement and the bulk period of the copolymer. In a physical confinement, symmetry breaking, confinement-induced entropy loss, structural frustration, and surface-segment energy are all able to significantly influence molecular organization, and thus create morphological diversity [12]. The effects of geometrical confinements have been studied at length in effort to identify efficient methods to obtain new long-range ordered structures. The most interesting system posited by previous research is the bulk lamella-forming block copolymer assembly between two solid walls (one-dimensional confinement). As far as diblock copolymers, considerable attention has been directed toward controlling lamellar orientation with respect to solid walls [13-15]. Self-assembled morphologies under one-dimensional confinement have not yet been sufficiently obtained in bulk systems, however.

Recently, researchers have endeavored to better understand the phase behavior of block copolymers in two-dimensional (2D) confinements. Two-dimensional (2D) confinement in the form of cylindrical pores with curved surfaces presents a more tightly confined geometry than that of thin films, and 2D confinements have been demonstrated to provide rich, varied structures. The use of cylindrical nanotubes produces 2D confinement with varying diameters for diblock copolymers as-studied in theory [16-18], simulation [19-25], and experiments [26-31]. Previous studies have also shown that the combined effects of confinement and curvature significantly influence the morphologies of copolymers in self-assembled systems. Yu et al. [12] used Monte Carlo simulation to study the self-assembly of block copolymers confined within cylindrical nanopores, for example. They found that the formation of self-assembly structures relied on pore diameter. For cylindrical-forming diblock copolymers, hexagonally-packed cylindrical domains were observed for large pores; in these domains, the symmetry and spacing of the cylinders was able to be altered by altering the shape and size of the pores [22]. Additionally, helical morphologies (as opposed to simple cylinders that are oriented 
parallel to the nanopores) have been observed in nanopores with smaller diameters [27,29]. Essentially, varying the diameters of nanotubes may be a suitable to control the conformations of copolymers within 2D confinement.

For 2D confined self-assembly, most studies focus on the phase behavior of copolymers confined in single-walled cylindrical nanotubes. The conformation of copolymers within multi-walled nanotubes, however, remains poorly understood. A multi-walled nanotube consists of concentrically-nested shells of single-walled nanotubes with different diameters [32]. A specialized nanotube, such as a multi-walled carbon nanotube (MWCNT), can potentially build robust and efficient nanomachines for future nanoelectromechanical systems, such as nanorotors [33,34], oscillators [35-37], and numerous other devices. Over the past few years, block copolymers have been utilized to modify the solution behavior of nanostructures within MWCNTs [38,39]. This suggests that internal structures can be controlled to enhance device performance. Copolymer nanocomposites represent a very interesting fundamental system for exploration. It has been established that enhancing the phase behavior of block copolymers self-assembled in multi-walled nanotubes is an important area of research. In one such study, Shvartzman-Cohen et al. [40] investigated the self-assembly of block copolymers (poly(ethylene oxide)-poly(propylene oxide)-poly(ethylene oxide)) in dispersions of multi-walled nanotubes as a function of temperature. Though several experimental studies have been reported in this area, the simulations of copolymers confined in multi-walled nanotubes have received relatively limited attention.

Computer simulation has been proven one of the most successful tools in predicting morphological behavior. Tight confinement of copolymers is expected to provide potential opportunities for manipulating novel morphologies, all of which can be successfully simulated by computer. Motivated by previous study, this study used molecular dynamics (MD) simulation to systematically investigate the self-assembled structures of block copolymers confined in multi-walled nanotubes with different diameters (the size of the nanotube is expected to fine-tune the self-assembled morphology of copolymers). This study is apparently the first to investigate the complex morphologies within multi-walled nanotubes using MD simulation to determine the morphology of the phase behavior of copolymers under the confinement of multi-walled nanotubes. This work provides a useful guide for future experiments that aim to fabricate different morphologies within nanotubes - the most fundamental and indispensable step toward realizing the application of copolymer self-assembly.

In addition to nanotube size, other significant effects of surface interaction on microdomain morphology have been validated in self-assembly systems both experimentally and theoretically. Previous studies have shown that surface preferential strength influences the orientation of the microdomains formed by each component. Wang et al. [41], for example, examined the dependence of self-assembled morphologies and structural parameters on degree of confinement and strength of surface preference. Using Monte Carlo simulations, $\mathrm{Yu}$ et al. investigated the influence of surface interaction on the self-assembled morphologies and chain conformations of a symmetric diblock copolymer confined in nanopores; such influence yielded a sequence of novel structures [42].

In this work, the self-assembled structures and phase diagram of diblock copolymers confined in multi-walled nanotubes are explored by gradually varying several system parameters over a wide range. Parameters include surface preferential strengths and confinement degrees. The primary goal of this work is two-fold: first, a detailed account of related studies, along with several unpublished 
results, is provided. The confinement-induced self-assembling mechanisms are then identified by systematically examining morphological transitions. Second, a preliminary understanding is built regarding the formation mechanism of self-assembled structures of block copolymers subjected to multi-walled nanotube confinement. The results of this study provide valuable theoretical guidance for the design of novel, polymeric nanomaterials under complex confinement.

\section{Model and Simulation Method}

\subsection{Modeling}

In the MD simulation, the model block copolymers confined in multi-walled nanotubes consist of A and B blocks of monomers. A detailed description of the model and algorithm is reviewed below. In this model, each chain consists of $N$ beads of identical size, which connect to form a linear diblock chain. The number of $\mathrm{A}$ and $\mathrm{B}$ monomers in the chain is $N_{A}=N_{B}=4$. The excluded volume interactions between the A and B block monomers are accounted for by the pure repulsion, cut, shift, and Lennard-Jones (LJ) potential, (often referred to as the Weeks Chandler Anderson potential [43]) expressed as follows:

$$
\begin{gathered}
u^{\mathrm{REP}}(r)=4 \varepsilon\left[\left(\frac{\sigma}{r}\right)^{12}-\left(\frac{\sigma}{r}\right)^{6}\right]+\varepsilon=u^{\mathrm{LJ}}(r)+\varepsilon, r \leq 2^{1 / 6} \sigma \\
\mathrm{u}^{\mathrm{REP}}(r)=0, r>2^{1 / 6} \sigma
\end{gathered}
$$

where $\sigma$ and $\varepsilon$ are the LJ parameters, and $r$ is the separation distance between beads. $\sigma, m$, and $\varepsilon$ are the length, mass, and energy units, respectively. In this paper, all other units are derived from these basic units, including temperature unit $T^{*}=\varepsilon / k_{\mathrm{B}}\left(k_{\mathrm{B}}\right.$ is Boltzmann constant) and time unit $\tau=\left(m \sigma^{2} / \varepsilon\right)^{1 / 2}$. Attractive potential between similar monomers (i.e., A-A or B-B) is used here to incorporate the physics of microphase separation between different blocks. Attractive potential was also used by Horsch et al. to model the equilibrium properties of block copolymer melts [44], which again involves LJ potential, but is cut and shifted at different values from those presented in Equation (1) as follows:

$$
\begin{gathered}
u^{\mathrm{ATT}}(r)=4 \varepsilon\left[\left(\frac{\sigma}{r}\right)^{12}-\left(\frac{\sigma}{r}\right)^{6}\right]-u^{\mathrm{LJ}}(2.5), r \leq 2.5 \sigma \\
u^{\mathrm{ATT}}(r)=0, r>2.5 \sigma
\end{gathered}
$$

The higher cutoff used above indicates that the interaction is not purely repulsive, and that monomers of the same type are attracted to one another. Horsch et al. [44] generated various points in the phase diagram with this potential, and found agreement between the phase diagram and mean field theory. Within a copolymer chain, the neighboring monomers are connected by a finitely extensible nonlinear elastic (FENE) potential [45]:

$$
u^{\mathrm{FENE}}(r)=-\frac{1}{2} k R_{\max }^{2} \ln \left[1-\left(\frac{r}{R_{\max }}\right)\right]
$$

where the maximum bond length is $R_{\max }=1.5 \sigma$, and the spring constant is $k=30 \varepsilon / \sigma^{2}$, as used by Kremer and Grest, to simulate polymer beads [45]. 


\subsection{Simulation Details}

To apply multi-walled nanotube confinement to the diblock copolymer, two cylindrical walls with different diameters, (with the same beads as those used in the polymeric bead-spring model) were constructed. The wall particles were fixed at their initially-defined positions and did not move with time. In this study, the ratio of the inside and outside radius $R_{\text {in }} / R_{\text {out }}$ was set to vary between $1 / 4$ and $1 / 2$. Interactions between the confining wall and copolymers with blocks A and B are represented by $\varepsilon_{\mathrm{WA}}$ and $\varepsilon_{\mathrm{WB}}$, respectively. Depending on the interactions between the tube-wall and the symmetric copolymer, differing strengths of surface preferences are considered: (1) The tube is strongly attractive to the majority blocks $\left(\varepsilon_{\mathrm{WA}}>\varepsilon_{\mathrm{WB}}\right)$; and (2) the tube is neutral $\left(\varepsilon_{\mathrm{WA}}=\varepsilon_{\mathrm{WB}}\right)$. The total monomer concentration in the system was maintained constantly at $\rho=0.8 \sigma^{-3}$. The nanotube axis falls along the $z$-direction, and the length of the nanotube is $z=30 \sigma$. The periodic boundary condition was applied to all three directions in all simulations. A dissipative particle dynamics (DPD) thermostat was employed to control the system temperature [46-48], and temperature $k_{\mathrm{B}} T$ was fixed at $1.2 \varepsilon / k_{\mathrm{B}}$. The friction coefficient was set to $\gamma=1.5 \tau^{-1}$. Simulations were run for an appropriate length of time until variables, such as pressure, potential energy, and mean squared end-to-end distance remained constant. All simulations were conducted with a time step of $\Delta t=0.005$. The initial system was equilibrated for $1 \times 10^{6}$ time steps before production runs were performed. Each production run ranged from $1.5 \times 10^{6}$ to $2 \times 10^{6}$ time steps.

\section{Results and Discussion}

This section first details the assemblies of $A_{4} B_{4}$ copolymers confined in single-walled nanotubes. The pairwise interaction parameters between blocks and nanotubes are $\varepsilon_{\mathrm{WA}}=0.7$ and $\varepsilon_{\mathrm{WB}}=0.1$. The outer radius of the outer nanotubes is $14 \sigma$ for both single-walled and multi-walled nanotubes. The typical equilibrium structure is presented in Figure 1. Relatively strong surface preference forced most B block monomers to contact the confining wall, whereas a few a monomers gathered in the pore center. The multi-cylindrical structures obtained in the above simulation are in accordance with structures predicted previously by $\mathrm{Yu}$ et al., who used a simulated annealing technique [20].

Next, the assembly of diblock copolymers within double-walled nanotubes with the same interaction parameters was simulated. The morphology shown in Figure 1b is different, accordingly, from that shown in Figure 1a. Notably, similar structures confined in the single-walled nanotubes were also found by Sevink et al. [49] and Wang et al. [50]. Obviously, both the incommensurability and the surface preference affect the formation of morphologies. These two factors cause the structure within the double-walled nanotubes to interact with the structure confined in the single-walled nanotube. No previous study has employed numerical simulation methods to focus on the phase behavior of self-assembled block copolymers within MWCNTs. There is similarly no previous experimentation regarding the conformation of copolymer assembled in nanotubes, due to the fact that it is exceedingly difficult to observe micro-size morphologies. Relevant studies [51] have, though, focused on the interaction between CNT and copolymers, and provided valuable information and compulsion for further research on the conformation transition of copolymers in MWCNTs. 


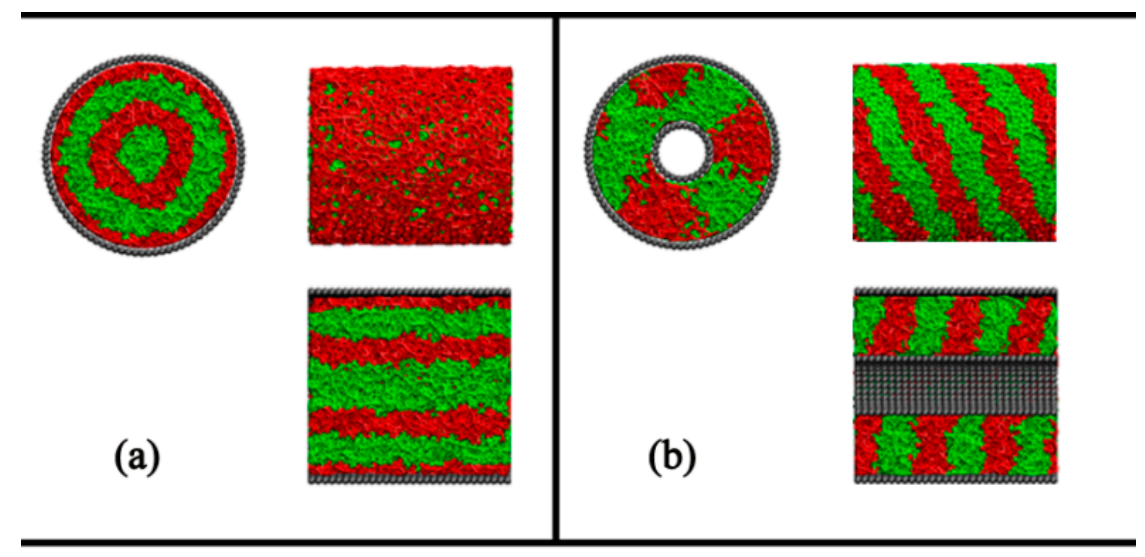

Figure 1. Morphologies of diblock copolymers within (a) a single nanotube and (b) multi-walled nanotubes. Top, side, and sectional views are shown. A blocks are shown in green, and B blocks in red.

A systematic study of surface preference-induced structures was conducted with different wall-polymer interactions, detailed as follows. Results are presented for two different cases, one in which the nanotube walls are more attractive to the A block than the B block, and another in which these walls are neutral to both A and B blocks.

Typical self-assembled morphologies in multi-walled nanotubes, as functions of different wall-polymer interactions, are summarized in Figure 2, in which the A blocks are shown in green and the B blocks in red. Quantitative measurement was used to determine the hierarchy of the morphologies. Three types of morphology were identified (Figure 2): (1) “ $\diamond$ " and " $\triangle$ " as 1-turn

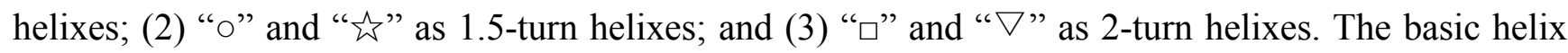
dimensions for the first morphology type are illustrated in Figure 3. $S_{1}$ is the linear length of one turn of the helix, and $R$ is the radius. If a single turn of the helix is stretched flat, the triangle shown on the right side of the figure is obtained, and $C=2 \pi R$ is the circumference of the turn. $L_{1}$ indicates the length of the string required to obtain a full turn, that is, $L_{1}=\sqrt{(2 \pi R)^{2}+\left(S_{1}\right)^{2}}$. The pitch of the helix is described by the tilted angle $\alpha$. The sine of $\alpha$ can be obtained from the relationship $\sin \alpha=\frac{L_{1}}{C}$. If $\sin \alpha<\frac{\sqrt{2}}{2}$, the morphology is type " $\triangle$ "; if $\sin \alpha \geq \frac{\sqrt{2}}{2}$, the morphology is type " $\diamond$ ".

In regard to the 1.5-turn and 2-turn helixes, the judgment method is the same as that used for the first morphology type (the red lines mark the top helix). Several new structures, including 1.5-turn and 2-turn helix morphologies, were discovered in this study. The surface preference parameter varied from 0.1 to 1.5 with a very small step, such that the selectivity of the confining surfaces changed from weakly preferential to strongly preferential toward the A block. When $\varepsilon_{\mathrm{WA}}=\varepsilon_{\mathrm{wB}}$, the multi-walled nanotubes were neutral. The sequences of morphologies (helical structures) were observed to be highly similar for the case of $\varepsilon_{\mathrm{WA}}=\varepsilon_{\mathrm{WB}}$. Notably, the transformation that occurs when $\varepsilon_{\mathrm{WA}}>\varepsilon_{\mathrm{WB}}$ is characterized by the morphology initially forming a helical structure, then forming a 1.5-turn helical structure (Figure 2). Increasing the attractive interaction between the confining wall and a copolymer blocks further results in 2-turn helical morphologies, which have never been observed either theoretically or experimentally in previous research. 


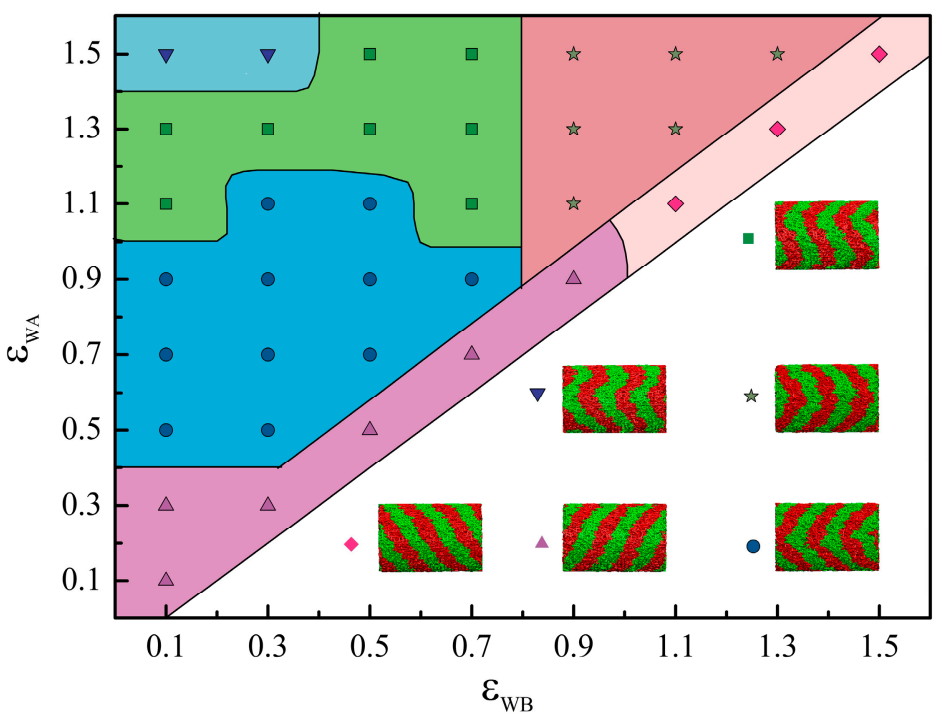

Figure 2. Phase diagram of copolymers under the confinement of a multi-walled nanotube system. Morphologies are organized as a function of surface preferences $\varepsilon_{\mathrm{WA}}$ and $\varepsilon_{\mathrm{WB}}$.

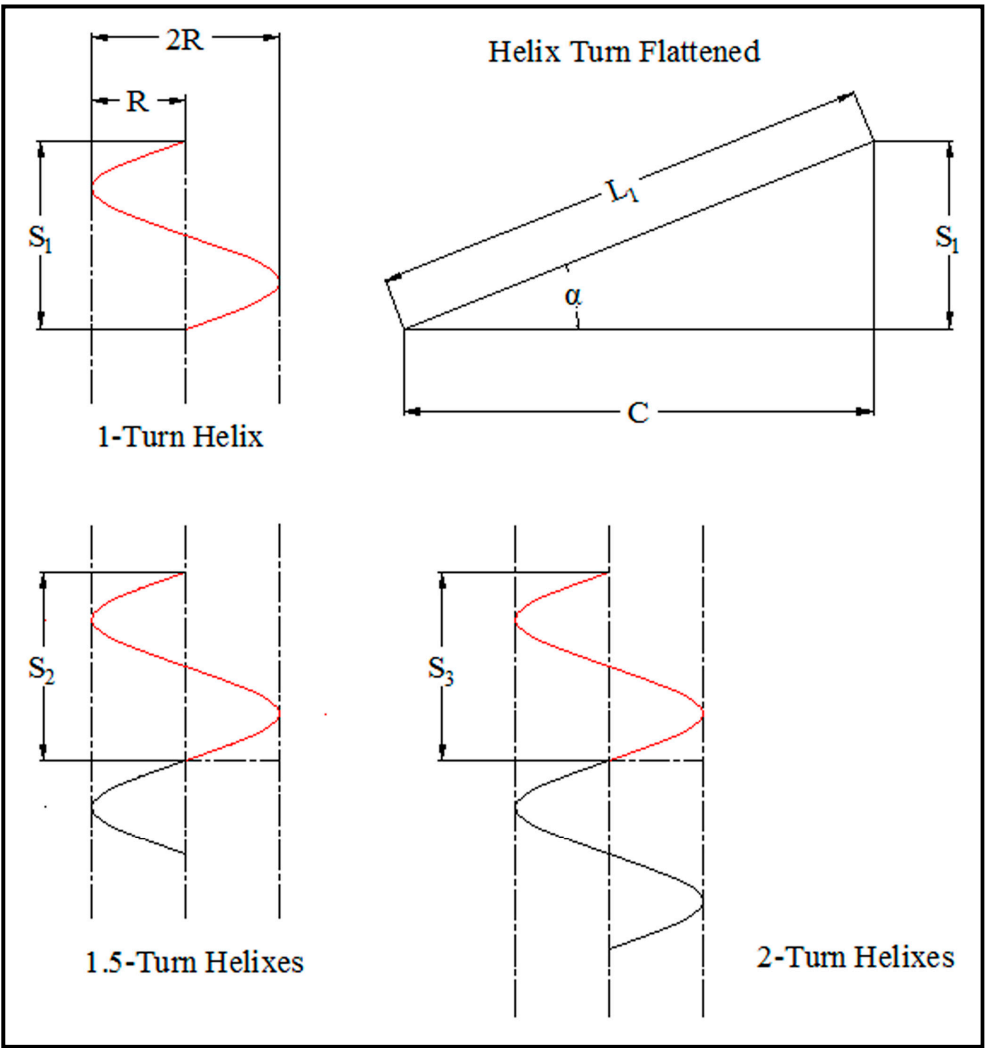

Figure 3. Basic dimensions of helixes.

Among these identified morphologies, helical lamellar structures present a common conformation when $\varepsilon_{\mathrm{WA}}=\varepsilon_{\mathrm{WB}}$, as shown in Figure 2. The interaction between the nanotubes and the blocks is a key parameter that determines the thickness of the helical lamellar structure. To describe the lamellar thickness quantitatively, the variations of the volume fractions of block copolymers are plotted in Figure 4. For the $\varepsilon_{\mathrm{WA}}=\varepsilon_{\mathrm{WB}}=0.7$ assembled system, the average thickness of the lamellar structure is $4.2 \sigma$, as shown in Figure 4a. However, in the case where $\varepsilon_{\mathrm{WA}}=\varepsilon_{\mathrm{WB}}=1.1$, the average thickness is 
$5.0 \sigma$. The thickness of the lamellar structure tends to increase with increase in surface preferential strength. The initial state of the system was an equilibrium-disordered state, suggesting that the A and B blocks were randomly distributed within the nanotubes. In the initial state, the block copolymers within the nanotubes were disordered. As the simulation progressed, the adsorption energy in the system increased and the morphologies gradually fell into order. When the interactions between the surfaces and the blocks were weak, the interactions among blocks A and B became the dominant factors, which determined the morphology. Given that blocks A and B are immiscible with each other, the two blocks tended to phase-separate into distinct domains and aggregate to form a small lamellar domain near each other. Cohesive and adsorption energies worked together to form the self-assembly conformation. If a stronger interaction exists between the nanotubes and the blocks, the adsorption energy serves a primary function. The helical lamellar structure of copolymers within nanotubes does not change with increase of adsorption strength in the case of $\varepsilon_{\mathrm{WA}}=\varepsilon_{\mathrm{WB}}$; however, the thickness of the lamellar does change.
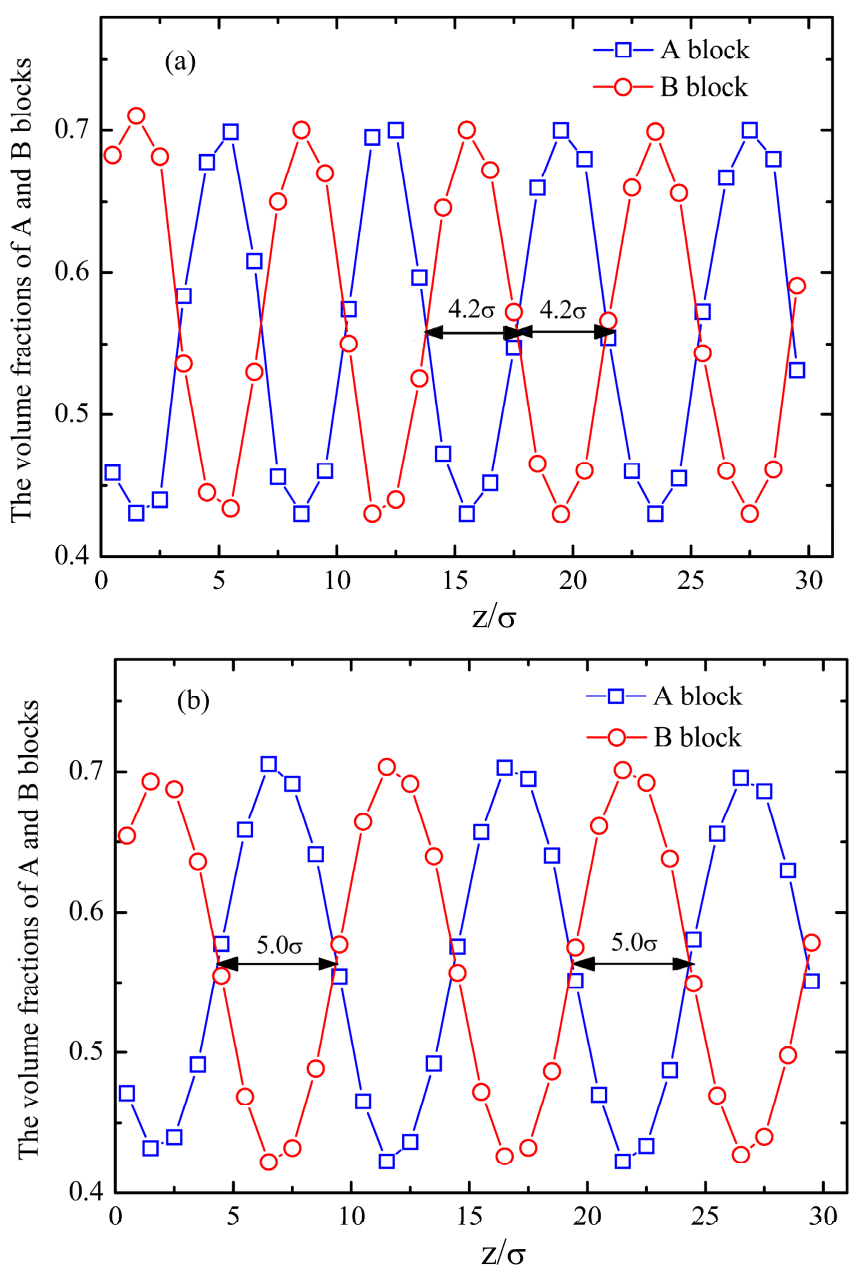

Figure 4. Variations of the volume fractions of blocks $\mathrm{A}$ and $\mathrm{B}$ along the $z$-direction for (a) $\varepsilon_{\mathrm{WA}}=\varepsilon_{\mathrm{WB}}=0.7$ and (b) $\varepsilon_{\mathrm{WA}}=\varepsilon_{\mathrm{WB}}=1.1$.

Another important factor that affects the morphologies of block copolymers confined in multi-walled nanotubes is nanotube size. Figure 5 shows the conformations of self-assembly at various values of $R_{\mathrm{in}} / R_{\mathrm{out}}$. During the simulation, two cases, $\varepsilon_{\mathrm{WA}}=\varepsilon_{\mathrm{WB}}$ and $\varepsilon_{\mathrm{WA}}>\varepsilon_{\mathrm{WB}}$, were considered. 
The sequence of morphologies for $\varepsilon_{\mathrm{WA}}=\varepsilon_{\mathrm{WB}}$ were very similar, where the conformations exhibited a weak dependence on the nanotube size $R_{\text {in }} / R_{\text {out }}$. Evidently, the helical lamellar structures in the assembled system form similarly to a packing mechanism. Given that the system is in a strongly segregated region, the block copolymers tend to maintain their lamellar structure. When $\varepsilon_{\mathrm{WA}}>\varepsilon_{\mathrm{WB}}$, though, the equilibrium morphology of the confined system is rather sensitive to nanotube size. As $R_{\text {in }} / R_{\text {out }}$ increased, the chains gradually stretched in the radial direction. The chain conformation was subsequently analyzed. Orientation and thickness of the lamellae are dependent on the size of the simulation box, even for periodic boundary conditions [52].

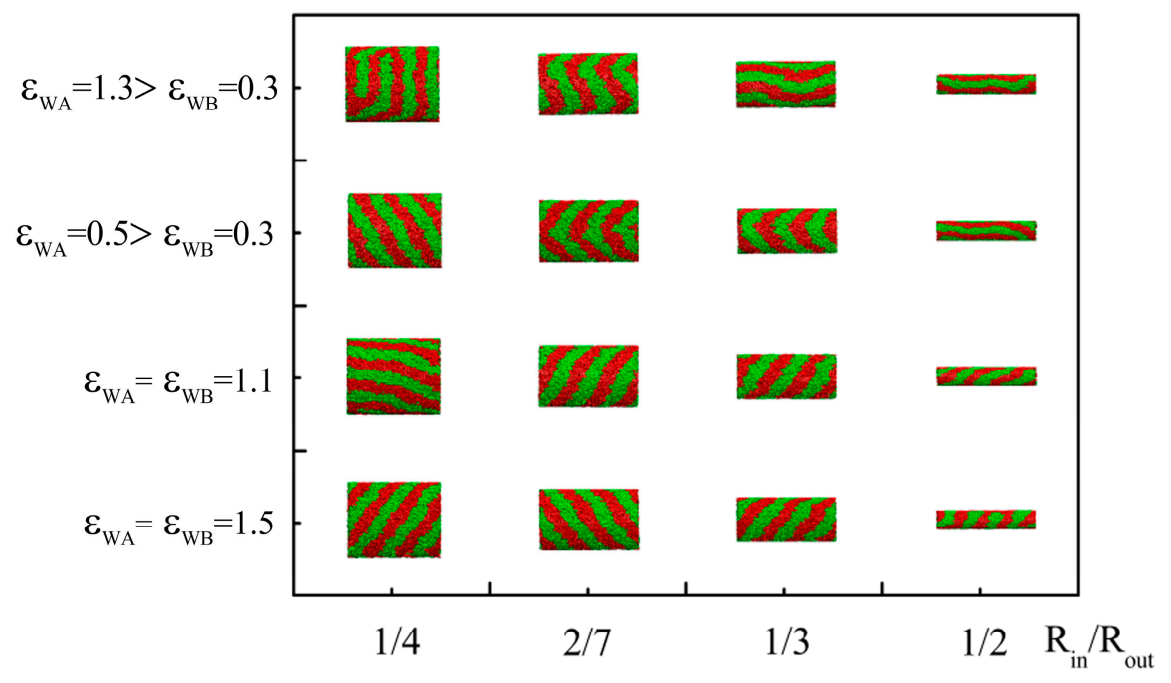

Figure 5. Self-assembled morphologies for multi-walled nanotube confined systems. Morphologies are organized as a function of the ratio $R_{\mathrm{in}} / R_{\text {out }}$ for different wall-polymer interactions.

To gain further insight into the confinement effects and surface preferences relative to chain conformation, the end-to-end distance of the chains was computed and plotted as a function of surface interaction as shown in Figure 6. For comparison, $\varepsilon_{\mathrm{WA}}>\varepsilon_{\mathrm{WB}}$ and $\varepsilon_{\mathrm{WA}}=\varepsilon_{\mathrm{WB}}$ were both analyzed. The end-to-end distance of copolymers is shown to increase with increasing interaction strength between the walls and chains (Figure 6a). Moreover, the end-to-end distance of copolymers with large $R_{\text {in }} / R_{\text {out }}$ is invariably less than those with smaller $R_{\text {in }} / R_{\text {out }}$. This demonstrates that the end-to-end distance under confinement increases with increasing wall-polymer interactions and outside diameter of multi-walled nanotubes, and that the blocks are in a stretched state. Figure 6a also shows the calculated sine tilted angle $\alpha$ for $\varepsilon_{\mathrm{WA}}=\varepsilon_{\mathrm{WB}}=1.0$, which increases with increase in outside diameter.

Under the assumption that attraction is stronger between the wall and the A block than the wall and the $\mathrm{B}$ block, the interaction between the wall and $\mathrm{B}$ block was fixed as $\varepsilon_{\mathrm{WB}}=0.1$. The end-to-end distance of copolymers initially decreased with increasing value of $\varepsilon_{\mathrm{WA}}$, then increased with further increase in the value of $\varepsilon_{\mathrm{WA}}$ when $R_{\mathrm{in}} / R_{\mathrm{out}}>1 / 4$. When $0.3<\varepsilon_{\mathrm{WB}}<0.7$, the end-to-end distance of the copolymers was considerably smaller, indicating that the chains were strongly compressed due to tight confinement. End-to-end distance increases as a result of increase in $\varepsilon_{\mathrm{WA}}$ and outer diameter $R_{\text {out }}$. This altogether indicates that morphological transitions are driven by the release of structural 
frustration in the form of stretching energy. One abnormal behavior, however, was exhibited where at the largest outer diameter $\left(R_{\text {in }} / R_{\text {out }}=1 / 4\right)$, the end-to-end distance of copolymers exhibited no significant change as interaction between the nanotubes and B block strengthened. This observation implies that the confinement effect weakens with increase in outer nanotube size, and that the effect of interaction strength is limited.
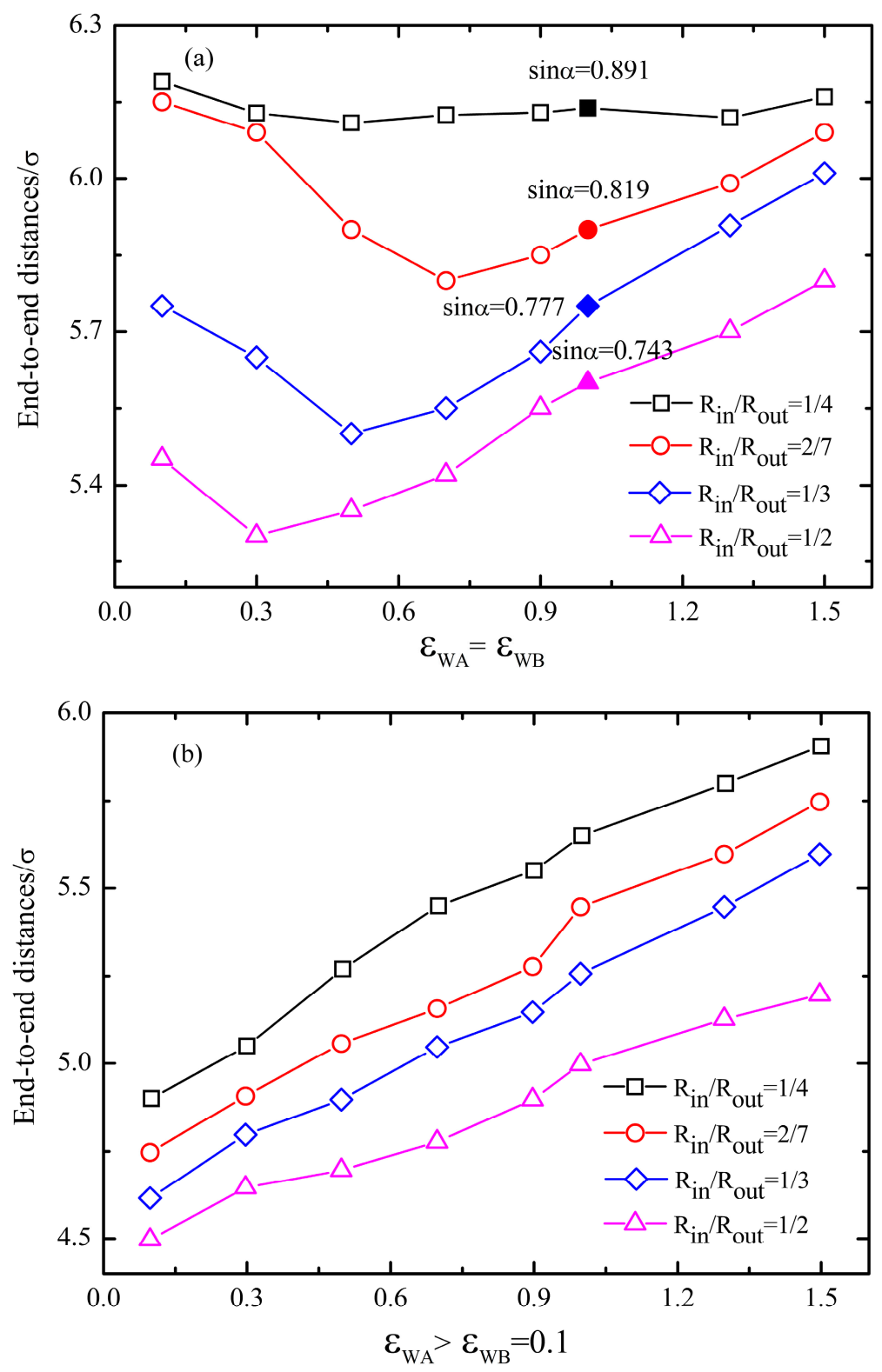

Figure 6. Variations in end-to-end distances of copolymers confined in multi-walled nanotubes when (a) $\varepsilon_{\mathrm{WA}}=\varepsilon_{\mathrm{WB}}$ or (b) $\varepsilon_{\mathrm{WA}}>\varepsilon_{\mathrm{WB}}=0.1$.

As shown in Figure 7, the thickness of helical lamellar structures in the self-assembled system, with equal surface preference for both the A and B blocks, is a function of interaction strength. The end-to-end distances of copolymers first decreased and then increased with increased interaction strength when $\varepsilon_{\mathrm{WA}}=\varepsilon_{\mathrm{WB}}$. When $\varepsilon_{\mathrm{WA}}>\varepsilon_{\mathrm{WB}}$, the thickness of the helical lamellar structure linearly increased with interaction strength for each size of multi-walled nanotube. The chains either compressed or stretched with varying adsorption strength. Free energy consists of three components in 
the system: (1) monomer-monomer interactions; (2) monomer-surface interactions; and (3) the entropic cost of stretching chains in segregated morphologies. The final morphologies the copolymers are the result of competition between these three contributions. (The end-to-end distance of chains as-measured was the third component, here).

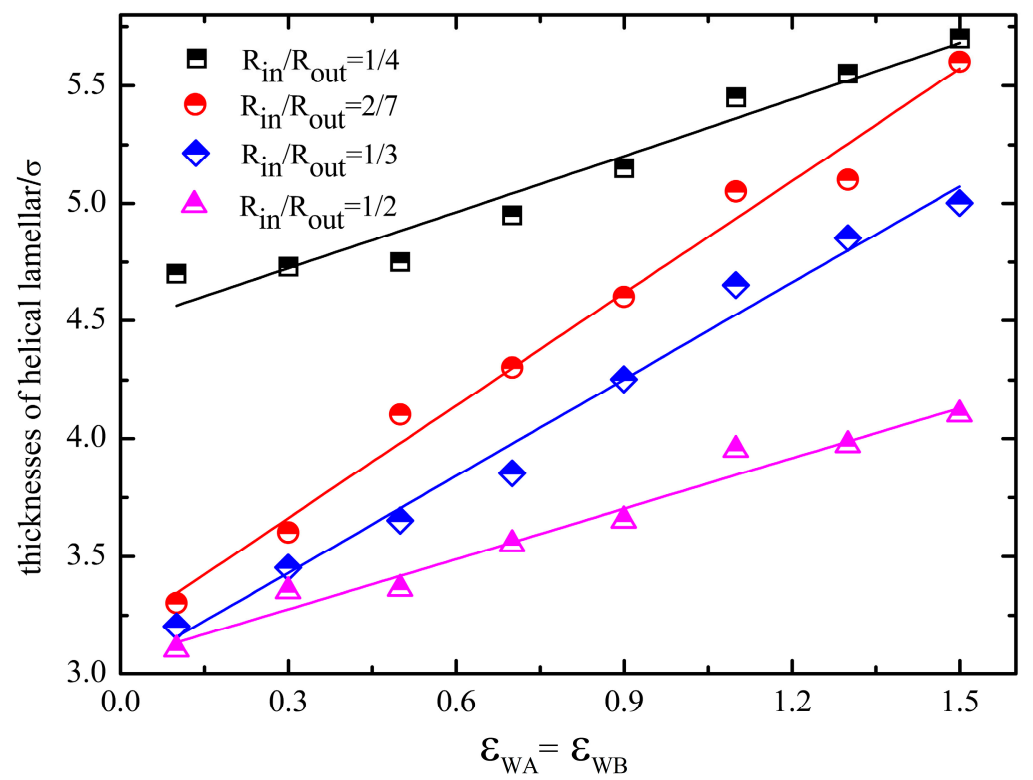

Figure 7. Thickness of helical lamella in morphologies as a function of the interaction strength between nanotubes and block copolymers.

\section{Conclusions}

This study employed an MD method to study the self-assembly of diblock copolymers confined in multi-walled nanotubes. Specific multi-walled cylindrical confinement is shown to result in a variety of novel morphologies. The size of multi-walled nanotubes and their surface preferences were systematically varied in order to examine their effects on the structures of various morphological chain conformations. A phase diagram was also plotted to illustrate that equilibrium morphology is largely controlled by the interaction strength between nanotube walls and block copolymers. Two representative cases were considered. The first of these takes a neutral wall $\left(\varepsilon_{\mathrm{WA}}=\varepsilon_{\mathrm{WB}}\right)$, where the influence of geometric confinement on the conformations of copolymers was significant, and the thickness of the helical lamellar structure increased with the interaction strength and size of confinement. In the other case, where the nanotubes strongly attracted block $\mathrm{A}\left(\varepsilon_{\mathrm{WA}}>\varepsilon_{\mathrm{WB}}\right)$, the confinement effect weakened with the increase in outer nanotube size $\left(R_{\text {in }} / R_{\text {out }} \leq 1 / 4\right)$. The dependence of chain conformation on the degree of confinement and the strength of the surface preferences is better clarified based on the results of this study. These findings can feasibly guide future research on locating and understanding nanostructures.

\section{Acknowledgments}

This work was supported by the National Natural Science Foundation of China (No. 51175223) and the Zhejiang Provincial Natural Science Foundation of China (Key Program, LZ14E050002). 


\section{Author Contributions}

This work is designed and performed by Yuxin Zuo and Ying Yu, under the supervision of Guoqiang Wang and Chuncheng Zuo. The other coauthors contributed in analysis of data, interpretation of the research finding and editing of this article.

\section{Conflicts of Interest}

The authors declare no conflict of interest.

\section{References}

1. Seul, M.; Andelman, D. Domain shapes and patterns: The phenomenology of modulated phases. Science 2007, 267, 476-483.

2. Li, W.H.; Liu, M.J.; Qiu, F.; Shi, A.C. Phase diagram of diblock copolymers confined in thin films. J. Phys. Chem. B 2013, 117, 5280-5288.

3. Park, M.; Harrison, C.; Chaikin, P.M.; Register, R.A.; Adamson, D.H. Block copolymer lithography: Periodic arrays of $\sim 10^{11}$ holes in 1 square centimeter. Science 1997, 276, 1401-1404.

4. Hamley, I.W. The Physics of Block Copolymers; Oxford University Press: Oxford, UK, 1998.

5. Okano, T. Biorelated Polymers and Gels: Controlled Release and Applications in Biomedical Engineering; Academic Press: Waltham, MA, USA, 1998.

6. Langner, K.M.; Sevink, G.J.A. Mesoscale modeling of block copolymer nanocomposites. Soft Matter 2012, 8, 5102-5118.

7. Shagolsem, L.S.; Sommer, J.U. Order and phase behavior of a cylinder forming diblock copolymers and nano-particles mixture in confinement: A molecular dynamics study. Macromol. Theor. Simul. 2011, 20, 329-339.

8. Fink, Y.; Winn, J.N.; Fan, S.; Chen, C.; Michel, J.; Joannopoulos, J.D.; Thomas, E.L. A dielectric omnidirectional reflector. Science 1998, 282, 1679-1682.

9. Bratko, D.; Chakraborty, A.K.; Shakhnovich, E.I. Frozen phases of random heteropolymers in disordered media. Phys. Rev. Lett. 1996, 76, 1844-1847.

10. Park, C.; Yoon, J.; Thomas, E.L. Enabling nanotechnology with self assembled block copolymer patterns. Polymer 2003, 44, 6725-6760.

11. Mezzenga, R.; Ruokolainen, J. Nanocomposites: Nanoparticles in the right place. Nat. Mater. 2009, 8, 926-928.

12. Yu, B.; Sun, P.; Chen, T.; Jin, Q.; Ding, D.; Li, B.; Shi, A.C. Confinement-induced novel morphologies of block copolymers. Phys. Rev. Lett. 2006, 96, doi:10.1103/PhysRevLett.96.138306.

13. Kellogg, G.J.; Walton, D.G.; Mayes, A.M.; Lambooy, P.; Russell, T.P.; Gallagher, P.D.; Satija, S.K. Observed surface energy effects in confined diblock copolymers. Phys. Rev. Lett. 1996, 76, 2503-2506.

14. Walton, D.G.; Kellogg, G.J.; Mayes, A.M.; Lambooy, P.; Russell, T.P. A free energy model for confined diblock copolymers. Macromolecules 1994, 27, 6225-6228.

15. Wang, Q.; Yan, Q.; Nealey, P.F.; de Pablo, J.J. Monte Carlo simulations of diblock copolymer thin films confined between two homogeneous surfaces. J. Chem. Phys. 2000, 112, 450-464. 
16. Ma, M.; Titievsky, K.; Thomas, E.L.; Rutledge, G.C. Continuous concentric lamellar block copolymer nanofibers with long range order. Nano. Lett. 2009, 9, 1678-1683.

17. Kalra, V.; Lee, J.H.; Park, J.H.; Marquez, M.; Joo, Y.L. Confined assembly of asymmetric block-copolymer nanofibers via multiaxial jet electrospinning. Small 2009, 5, 2323-2332.

18. Ma, M.; Thomas, E.L.; Rutledge, G.C.; Yu, B.; Li, B.; Jin, Q.; Shi, A.C. Gyroid-forming diblock copolymers confined in cylindrical geometry: A case of extreme makeover for domain morphology. Macromolecules 2010, 43, 3061-3071.

19. Shin, K.; Xiang, H.; Moon, S.I.; Kim, T.; McCarthy, T.J.; Russell, T.P. Curving and frustrating flatland. Science 2004, 306, doi:10.1126/science.1100090.

20. Yu, B.; Sun, P.; Chen, T.; Jin, Q.; Ding, D.; Li, B.; Shi, A.C. Self-assembly of diblock copolymers confined in cylindrical nanopores. J. Chem. Phys. 2007, 127, doi:10.1063/1.2768920.

21. Pinna, M.; Guo, X.; Zvelindovsky, A.V. Diblock copolymers in a cylindrical pore. J. Chem. Phys. 2009, 131, doi:10.1063/1.3264946.

22. Xiang, H.; Shin, K.; Kim, T.; Moon, S.I.; McCarthy, T.J.; Russell, T.P. Block copolymers under cylindrical confinement. Macromolecules 2004, 37, 5660-5664.

23. Yu, B.; Jin, Q.; Ding, D.; Li, B.; Shi, A.C. Confinement-induced morphologies of cylinder-forming asymmetric diblock copolymers. Macromolecules 2008, 41, 4042-4054.

24. Shagolsem, L.S.; Sommer, J.U. Order and phase behavior of thin film of diblock copolymer-selective nanoparticle mixtures: A molecular dynamics simulation study. Macromolecules 2014, 47, 830-839.

25. Wu, X.; Chen, P.; Feng, X.; Xia, R.; Qian, J. Effect of selective nanoparticles on phase separation of copolymer-nanoparticle composites confined between two neutral surfaces. Soft Matter 2013, 9 , 5909-5915.

26. Yang, Y.; Qiu, F.; Zhang, H.; Yang, Y. Cylindrical phase of diblock copolymers confined in thin films. A real-space self-consistent field theory study. Polymer 2006, 47, 2205-2216.

27. Xiang, H.; Shin, K.; Kim, T.; Moon, S.; McCarthy, T.J.; Russell, T.P. The influence of confinement and curvature on the morphology of block copolymers. J. Polym. Sci. B Polym. Phys. 2005, 43, 3377-3383.

28. Heckmann, M.; Drossel, B. Strong stretching theory for diblock copolymers in thin films. J. Chem. Phys. 2008, 129, doi:10.1063/1.3027437.

29. Xiang, H.; Shin, K.; Kim, T.; Moon, S.I.; McCarthy, T.J.; Russell, T.P. From cylinders to helices upon confinement. Macromolecules 2005, 38, 1055-1056.

30. Dobriyal, P.; Xiang, H.; Kazuyuki, M.; Chen, J.T.; Jinnai, H.; Russell, T.P. Cylindrically confined diblock copolymers. Macromolecules 2009, 42, 9082-9088.

31. Jang, S.G.; Khan, A.; Hawker, C.J.; Kramer, E.J. Morphology evolution of PS- $b$-P2VP diblock copolymers via supramolecular assembly of hydroxylated gold nanoparticles. Macromolecules 2012, 45, 1553-1561.

32. Iijima, S. Helical microtubules of graphitic carbon. Nature 1991, 354, 56-58.

33. Bourlon, B.; Glattli, D.C.; Miko, C.; Forró, L.; Bachtold, A. Carbon nanotube based bearing for rotational motions. Nano Lett. 2004, 4, 709-712.

34. Fennimore, A.M.; Yuzvinsky, T.D.; Han, W.Q.; Fuhrer, M.S.; Cumings, J.; Zettl, A. Rotational actuators based on carbon nanotubes. Nature 2003, 424, 408-410.

35. Forro, L. Beyond gedanken experiments. Science 2000, 289, 560-561. 
36. Legoas, S.B.; Coluci, V.R.; Braga, S.F.; Coura, P.Z.; Dantas, S.O.; Galvao, D.S. Molecular-dynamics simulations of carbon nanotubes as gigahertz oscillators. Phys. Rev. Lett. 2003, 90, doi:10.1103/PhysRevLett.90.055504.

37. Zheng, Q.; Jiang, Q. Multiwalled carbon nanotubes as gigahertz oscillators. Phys. Rev. Lett. 2002, 88, doi:10.1103/PhysRevLett.88.045503.

38. Szleifer, I.; Yerushalmi-Rozen, R. Polymers and carbon nanotubes-Dimensionality, interactions and nanotechnology. Polymer 2005, 46, 7803-7818.

39. Shin, H.I.; Min, B.G.; Jeong, W.; Park, C. Amphiphilic block copolymer micelles: New dispersant for single wall carbon nanotubes. Macromol. Rapid Commun. 2005, 26, 1451-1457.

40. Shvartzman-Cohen, R.; Florent, M.; Goldfarb, D.; Szleifer, I.; Yerushalmi-Rozen, R. Aggregation and self-assembly of amphiphilic block copolymers in aqueous dispersions of carbon nanotubes. Langmuir 2008, 24, 4625-4632.

41. Wang, Z.; Li, B.; Jin, Q.; Ding, D.; Shi, A.C. Simulated annealing study of self-assembly of symmetric ABA triblock copolymers confined in cylindrical nanopores. Macromol. Theory Simul. 2008, 17, 86-102.

42. Yu, B.; Li, B.; Jin, Q.; Ding, D.; Shi, A.C. Self-assembly of symmetric diblock copolymers confined in spherical nanopores. Macromolecules 2007, 40, 9133-9142.

43. Weeks, J.D.; Chandler, D.; Andersen, H.C. Role of repulsive forces in determining the equilibrium structure of simple liquids. J. Chem. Phys. 1971, 54, 5237-5247.

44. Horsch, M.A.; Zhang, Z.; Iacovella, C.R.; Glotzer, S.C. Hydrodynamics and microphase ordering in block copolymers: Are hydrodynamics required for ordered phases with periodicity in more than one dimension? J. Chem. Phys. 2004, 121, 11455-11462.

45. Kremer, K.; Grest, G.S. Dynamics of entangled linear polymer melts: A molecular-dynamics simulation. J. Chem. Phys. 1990, 92, 5057-5086.

46. Soddemann, T.; Dünweg, B.; Kremer, K. Dissipative particle dynamics: A useful thermostat for equilibrium and nonequilibrium molecular dynamics simulations. Phys. Rev. E 2003, 68, doi:10.1103/PhysRevE.68.046702.

47. Hoogerbrugge, P.J.; Koelman, J.M.V.A. Simulating microscopic hydrodynamic phenomena with dissipative particle dynamics. Europhys. Lett. 1992, 19, 155-160.

48. Español, P.; Warren, P. Statistical mechanics of dissipative particle dynamics. Europhys. Lett. 1995, 30, 191-196.

49. Sevink, G.J.A.; Zvelindovsky, A.V.; Fraaije, J.G.E.M.; Huinink, H.P. Morphology of symmetric block copolymer in a cylindrical pore. J. Chem. Phys. 2001, 115, doi:10.1063/1.1403437.

50. Wang, Q. Symmetric diblock copolymers in nanopores: Monte Carlo simulations and strongstretching theory. J. Chem. Phys. 2007, 126, doi:10.1063/1.2406078.

51. Zou, P.; Shi, G.Y.; Pan, C.Y. Large-compound vesicle-encapsulated multiwalled carbon nanotubes: A unique route to nanotube composites. J. Polym. Sci. A Polym. Chem. 2009, 47, 3669-3679.

52. Nardai, M.M.; Zifferer, G. Concentration dependence of size, shape, and orientation of copolymers, 1-Linear diblock and 4-arm hetero star polymers. Macromol. Theory Simul. 2011, 20, 675-689.

(C) 2015 by the authors; licensee MDPI, Basel, Switzerland. This article is an open access article distributed under the terms and conditions of the Creative Commons Attribution license (http://creativecommons.org/licenses/by/4.0/). 